\title{
Electron Microscopy Characterization of Fe-Ga and Fe-Si-B Fine Particles
}

\author{
Virgil C. Solomon, ${ }^{*}$ David J. Smith, ${ }^{* *}$ Jung-Il Hong, ${ }^{* * *}$ and Ami E. Berkowitz**** \\ * Institute of Materials Science, University of Connecticut, Storrs, CT 06269-3136 \\ ** Department of Physics, Arizona State University, Tempe, AZ 85287-1504 \\ *** School of Materials Science \& Engineering, Georgia Institute Technology, Atlanta, GA 30126 \\ **** Center for Magnetic Recording Research and Department of Physics, University of California \\ - San Diego, La Jolla, CA 92093
}

Fine particles of Fe-Ga and Fe-Si-B alloys have been obtained by spark-erosion using different dielectrics. The particles are spherical in shape with the average diameter ranging from a few nanometers to tenths of micrometers, as a function of the process parameters (sparking energy, apparatus configuration, dielectrics [1]). In the present work Fe-Ga particles prepared in liquid nitrogen or liquid argon, as dielectrics, and Fe-Si-B particles made in water or water/ethanol (50/50 by volume) have been investigated using analytical scanning and transmission electron microscopy methods in a FEI XL30 FEG SEM equipped with an EDAX-TSL Orientation Imaging Microscopy (OIM) system and a JEOL JEM-2010 S/TEM, respectively. The cross-sectioned samples for EM investigation were prepared in a FEI Dual Beam Nova 200 NanoLab focused ion beam machine.

Fe-Ga alloy is regarded as the archetype of giant field-induced magnetostrictive materials. For bulk single crystalline Fe-(15-20 at. \%)Ga samples, the magnetostriction is about ten times larger than that of pure $\mathrm{Fe}\left(\lambda_{111}=21 \times 10^{-6}\right)$, and depends on the thermal history of the alloy. However, the largest magnetostriction has been reported in melt-spun Fe-Ga alloy, $\lambda=2100 \times 10^{-6}$. Therefore, it is expected that a technique in which single crystals of $\mathrm{Fe}-\mathrm{Ga}$ alloys are obtained by rapid quenching may provide a direct way to obtain giant magnetostrictive materials. In the spark-erosion process sub- and micrometer sized Fe-19 at \% Ga particles are rapidly cooled in situ at quench rates in excess of $10^{9}$ $\mathrm{K} / \mathrm{s}$ [1]. The SEM investigation of cross-sectioned samples, prepared using FIB, revealed that approximately $80 \%$ of the particles, with average sizes between $20-25 \mu \mathrm{m}$, were single crystals. Figure 1 shows typical OIM investigation results for these particles. The electron backscattering diffraction patterns were indexed using the bcc crystal structure of $\alpha$-Fe (A2, Im3m, a = 2.905 $\AA$ ). Composite materials made by mixing these particles with epoxy had shown magnetostriction values similar to those reported for bulk polycrystalline Fe-Ga alloys [2].

Ferromagnetic Fe-Si-B particles are materials of practical interest for soft magnetic applications. In order to reduce eddy-current losses, it is desirable that the particles are coated with a continuous electrically insulating layer. The purpose of this work was to investigate the presence of an oxide layer on the surfaces of Fe-Si-B particles, Fig. 2. The existence of a continuous thin oxide layer at the particle surfaces was demonstrated by EDX/WDS investigation. In order to determine both the composition and the film thickness, the typical analytical thin film on a substrate approach [3] was employed. Figures 2(c) to (e) show the EDX spectra taken from the same point of the sample for beam energies of $20 \mathrm{keV}, 5 \mathrm{keV}$ and $2 \mathrm{keV}$. As shown in Fig. 2(e), a beam energy $\left(\mathrm{E}_{0}\right)$ of $2 \mathrm{keV}$ was enough to excite the characteristic X-ray peak of oxygen, since the overvoltage $U=E_{0} / E_{c}=3.8241$, where $E_{c}=0.523 \mathrm{keV}$ is the critical ionization energy of the oxygen $\mathrm{K}_{\alpha}$ shell. The thickness of the oxide layer was less than $100 \mathrm{~nm}$, as measured experimentally using both STEM investigations of cross-sectioned particles, Fig. 2(f), and high-resolution Auger electron spectroscopy. Monte Carlo calculations supported the experimental evidence. The average oxygen concentration in the surface layer was about 10 at. \%, and decreased from the exterior toward the interior of the sample [4]. 


\section{References}

[1] A. E. Berkowitz and J. L. Walter, J. Mater. Res. 2 (1987) 277.

[2] J. I. Hong et al., Appl. Phys. Lett. 89 (2006) 142506.

[3] J. Goldstein et al. Scanning Electron Microscopy and X-Ray Microanalysis, ${ }^{\text {rd }}$ ed., Springer Science, New York, 2003.

[4] The use of facilities in the John M. Cowley Center for High Resolution Electron Microscopy at Arizona State University is gratefully acknowledged.
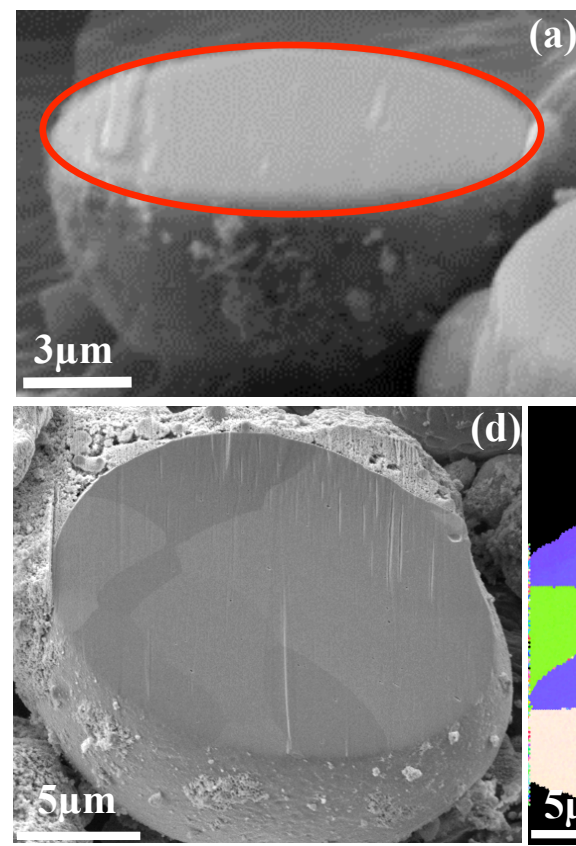
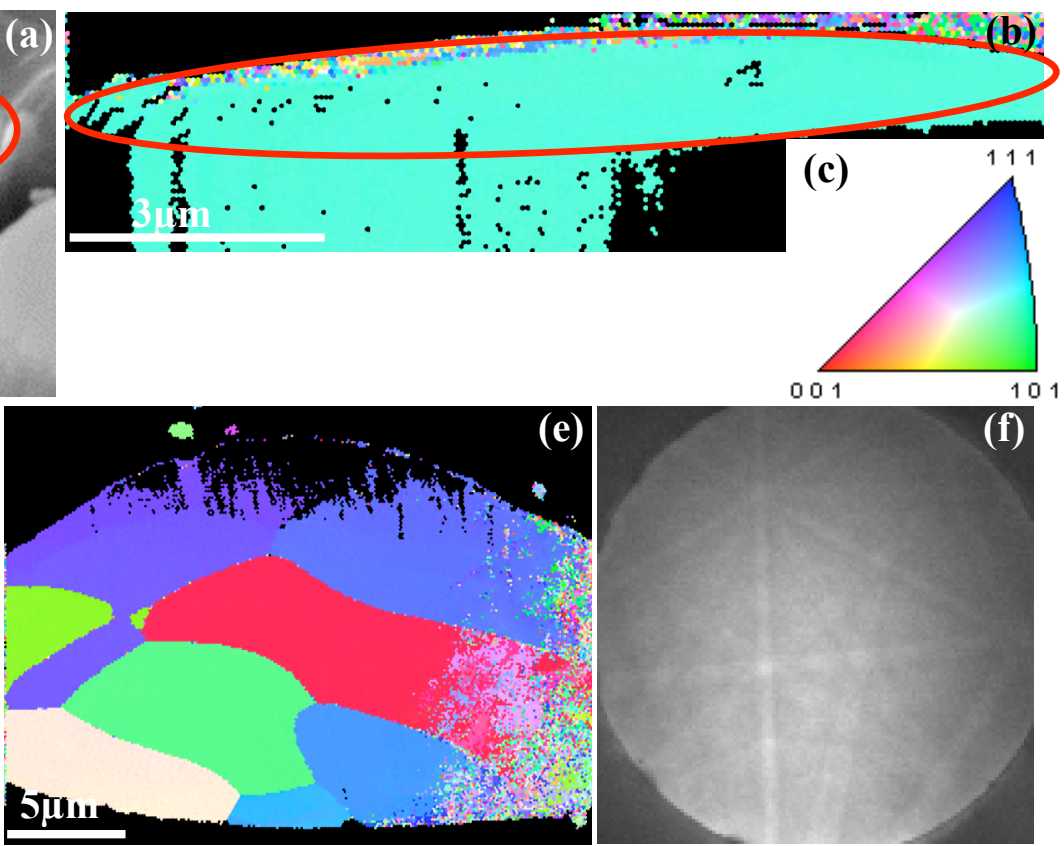

(f)

Fig. 1 (a), (d) Scanning ion micrographs of FIB cross-sectioned single and polycrystalline Fe-Ga particles spark-eroded in liquid argon; (b), (e) Inverse Pole Figure (IPF) maps from (a) and (d), respectively. The colors correspond to the crystal orientations as shown in (c), the stereographic cubic (001) projection. (f) Electron backscattering diffraction pattern from the particle in (d).
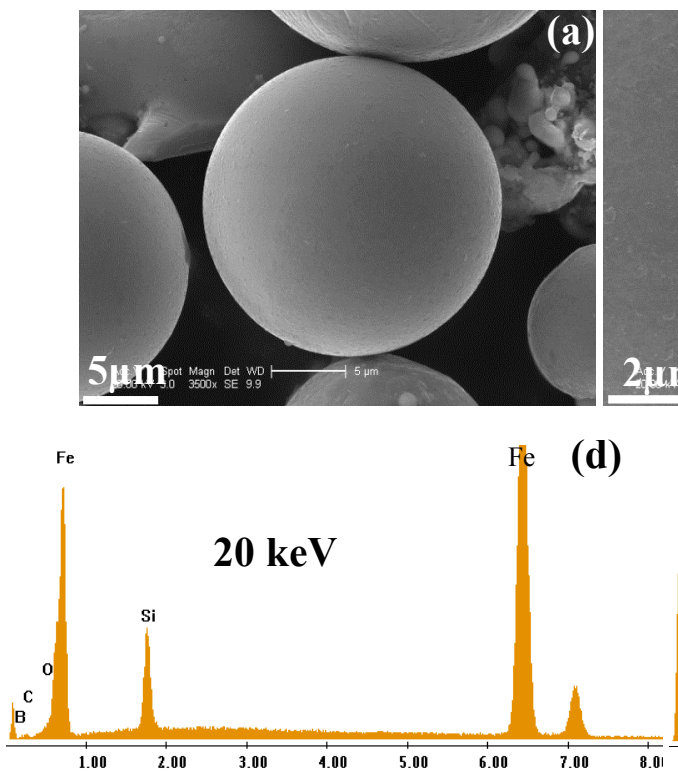

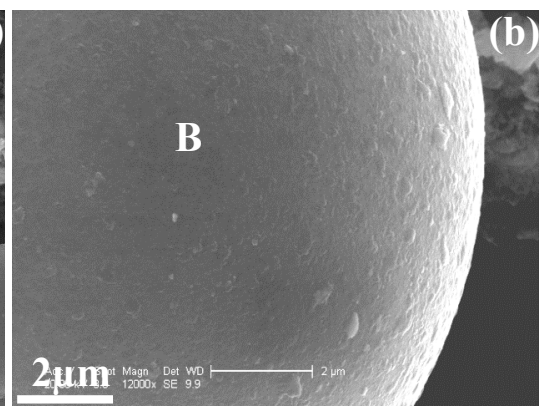

(e)
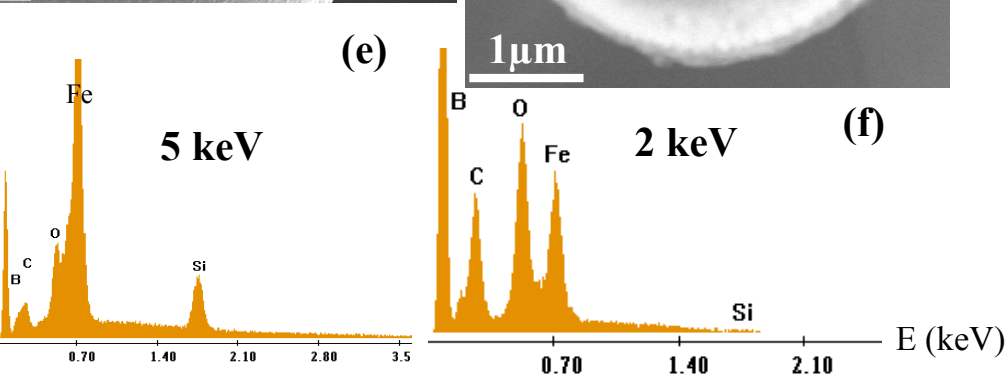

Fig. 2 (a), (b) Typical morphology of Fe-Si-B particles spark-eroded in water. (c) STEM dark-field micrograph of cross-sectioned particle. (d)-(f) EDX spectra taken at different beam energies from the area in (b) labeled B. 